\title{
X-Ray Scattering and its Benefits for X-Ray Spectrometry at the SEM
}

\author{
V.-D. Hodoroaba, ${ }^{*}$ V. Rackwitz, ${ }^{*}$ and D. Reuter* \\ * Federal Institute for Materials Research \& Testing (BAM), D-12205 Berlin, Germany
}

In recent years an increasing number of micro-focus X-ray sources have been used in combination with an energy dispersive spectrometer (EDS) at the scanning electron microscope (SEM) [1]. The main improved analytical figure of merit of the X-ray fluorescence analysis (XRFA) is significantly lower limits of detection in comparison to those of electron probe microanalysis (EPMA).

One particular analytical challenge of this combination (electron and photon excitation) consists of the accurate determination of elements of mid and high atomic number at trace level concentrations contained in light matrices such as glass, ceramic, polymer, etc. The background reconstruction in the XRF spectra for this type of samples is difficult and for "complicated" samples constitutes a source of uncertainties in the determination of net peak areas necessary for the quantification (see Fig. 1 for the two quantification software packages iMOXS-Quant and WinAxil). The procedures applied for the background reconstruction are of mathematical and empirical nature. Based on the modeling of X-ray scattering (as the main contribution to the prominent spectral background) first principles of a physical model for the calculation of the background will be presented. Hence, predictions of attainable detection limits are also possible.

By changing the components of the X-ray source (either the X-ray tube with a different target material or even the X-ray optics) the excitation of the analytes can be optimized. However, the real transmission of the X-ray optics suffers quite sensitively from unavoidable small misalignments of the X-ray spot on the tube target relative to the X-ray optics. A procedure for the determination of the real transmission of the X-ray optics relying on the calculation of scatter spectra is described [2].

Of decisive importance for the standardless quantification with ED-EPMA and ED-XRFA is the exact knowledge of the ED spectrometer efficiency. A new procedure for the calibration of an EDS up to $50 \mathrm{keV}$ will be presented by using both excitations with electrons and photons at the SEM. A calibrated detector [3] and reference materials especially designed/chosen for this purpose are necessary. In the high-energy range one takes advantages of the intensive X-ray scattering on materials with low atomic number.

References

[1] M. Procop, V.-D. Hodoroaba, Microchim. Acta 161 (2008) 413.

[2] V.-D. Hodoroaba, M. Procop, X-Ray Spectrom. in press.

[3] F. Scholze, B. Beckhoff, M. Kolbe, M. Krumrey, M. Müller, G. Ulm, Microchim. Acta 155 (2006) 275.

[4] This work is partly supported by a "MNPQ-Transfer" project (BMWi, 23/07) and the "BAMInnovationsoffensive" program, respectively. 

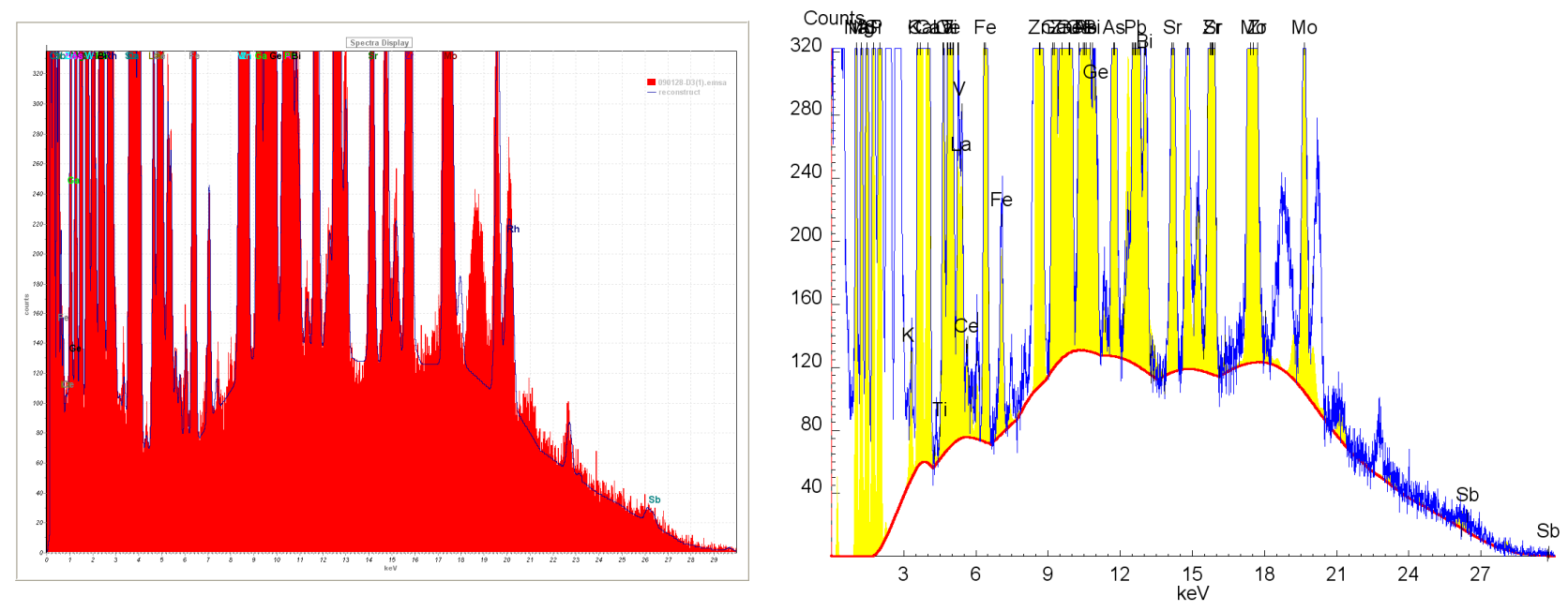

FIG. 1. Example of XRF spectrum of a glass CRM ("BR D3", Breitländer GmbH, Hamm, Germany) and two background models (left iMOXS-Quant and right WinAxil). 